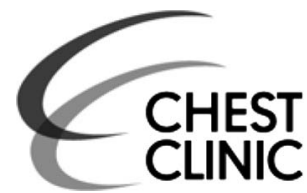

${ }^{1}$ Pathology Unit, University Hospital Policlinico of Modena, Modena, Italy

${ }^{2}$ Pathology Unit, Azienda USL Valle d'Aosta, Hospital "Parini", Aosta, Italy

${ }^{3}$ Unit of Oncology, University Hospital of Parma, Parma, Italy ${ }^{4}$ Department of Oncology, University Hospital Policlinico of Modena, Modena, Italy ${ }^{5}$ Unit of Oncology, Hospital of Sassuolo, Modena, Italy ${ }^{6}$ Unit of Thoracic Surgery, Arcispedale Santa Maria NuovaIRCCS of Reggio Emilia, Reggio Emilia, Italy

Correspondence to Dr Filippo Lococo, Unit of Thoracic Surgery, Arcispedale Santa Maria Nuova-IRCCS of Reggio Emilia, via Risorgimento 80, Reggio Emilia 42100, Italy: filippo.lococo@asmn.re.it

Received 12 September 2016 Revised 26 October 2016 Accepted 12 November 2016 Published Online First 2 December 2016

\title{
'Turban-like' skull metastasis from pulmonary adenocarcinoma
}

\author{
Maria Cecilia Mengoli, ${ }^{1}$ Giulio Rossi, ${ }^{2}$ Marcello Tiseo, ${ }^{3}$ Fausto Barbieri, ${ }^{4}$ Micol Pifferi, ${ }^{5}$ \\ Federica Bertolini, ${ }^{4}$ Filippo Lococo ${ }^{6}$
}

A 55-year-old woman presented with a progressive psychomotor impairment. She spoke slowly and her motor retardation interfered with activities of daily living. The patient reported that 5 months earlier, she had experienced blunt force trauma of vertex with a progressive swelling of the head. At the time of presentation, a painful, fixed and hard lesion was detected at palpation. Encephalic CT and MRI revealed an osteolytic mass of the skull of $10 \mathrm{~cm}$ in maximum diameter, infiltrating the left frontoparietal bones with calcifications (figure 1A, B). The lesion caused a mass effect, without infiltration of the brain parenchyma. A whole-body CT discovered a $4 \mathrm{~cm}$ pulmonary lesion of the left upper lobe (figure 1C). The biopsy (figure 2) of the skull mass revealed a metastatic pulmonary adenocarcinoma (thyroid transcription factor 1 positive, $\mathrm{p} 40 / \Delta \mathrm{Np} 63$ negative). Molecular analysis disclosed a sensitising exon 19 (delE746-A750) epidermal growth factor receptor (EGFR) gene mutation. ALK and ROS-1 rearrangements were negative. An EGFR inhibitor (erlotinib, $150 \mathrm{mg}$ once daily) was started. The women experienced a significant clinical benefit with a slight dimensional reduction of the pulmonary lesion (stable disease according to revised RECIST guidelines ${ }^{1}$ ) while the skull metastasis was stable with an increased density at 8 months follow-up.

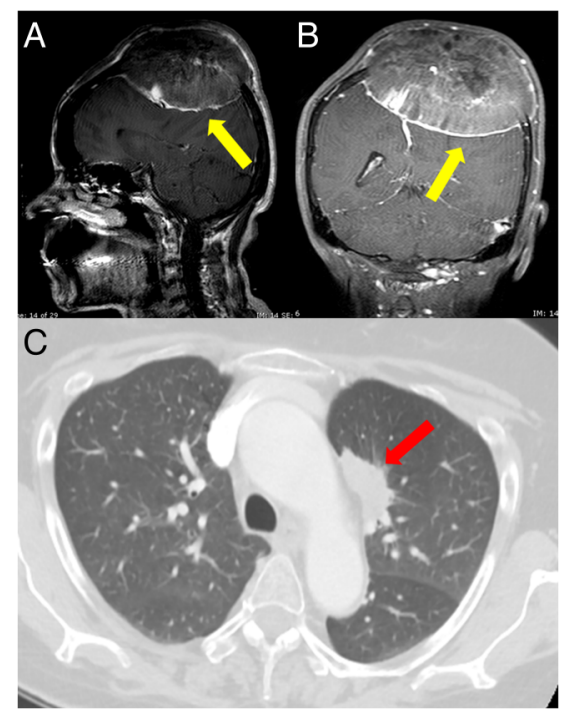

Figure 1 Brain CT scan showed the $10 \mathrm{~cm}$ skull mass infiltrating left frontoparietal bones with concurrent calcifications ( $A$ and $B$, yellow arrow). Thoracic $C T$ scan disclosed a $4 \mathrm{~cm}$ lesion of the left upper lobe with peripheral ground glass opacities (C, red arrow).

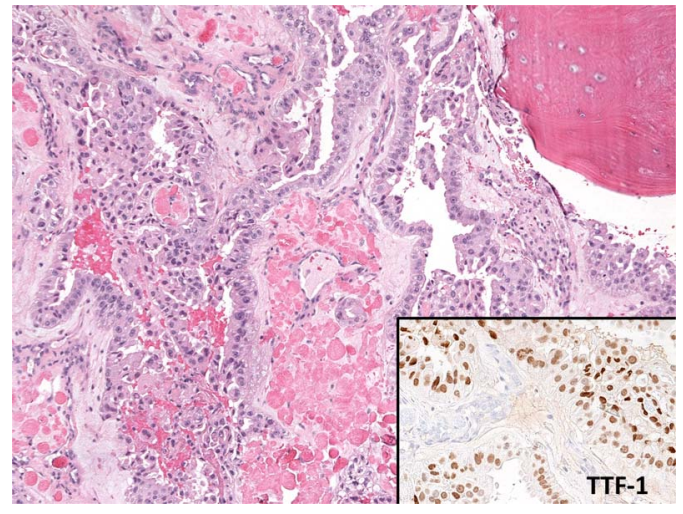

Figure 2 Histological examination (H\&E) of the skull lesion revealed an adenocarcinoma with glandular pattern of growth (on the left) infiltrating bone (on the right) with nuclear expression of thyroid transcription factor 1 (TTF-1) at immunohistochemistry (inset) consistent with metastatic lung adenocarcinoma to the skull.

In dealing with a skull mass, the first differential diagnosis is between primary and metastatic neoplasms of various cell lineages, including soft tissue tumours, haematologic proliferations, brain tumours and metastasis from several neoplasms. ${ }^{2}$ A close correlation between clinical and radiological data together with morphological and immunohistochemical stains on biopsy is mandatory. ${ }^{2}$ Among carcinomas, skull metastases are mostly noted in hepatocellular carcinoma, cholangiocarcinoma, prostate, thyroid and breast cancers. ${ }^{2}$ Although the rate of bone metastases associated with lung carcinoma is estimated at $36 \%$, only $3 \%$ of lung cancers metastasise to skull. ${ }^{3}$ Skull metastases are usually diagnosed as multiple lesions in the context of a known primary tumour. ${ }^{2}$ Rarely do they represent the first single manifestation of an unknown carcinoma, ${ }^{4}$ and lung adenocarcinoma presenting as skull mass is exceedingly rare. ${ }^{3}$ No significant relationship has been demonstrated between EGFR mutation and this unusual metastatic pattern.

Contributors MCM conceived and wrote the manuscript. GR performed the pathological evaluation. MT performed the literature review. FB supported in the comprehension of the oncological aspects. MP collected radiological imaging. FB was involved in the oncological cares of the patient. FL wrote along with MCM the manuscript.

Competing interests None declared.

Patient consent Obtained.

Provenance and peer review Not commissioned; externally peer reviewed. 


\section{REFERENCES}

1 Eisenhauer EA, Therasse $P$, Bogaerts J, et al. New response evaluation criteria in solid tumours: revised RECIST guideline (version 1.1). Eur J Cancer 2009;45:228-47.

2 Colas L, Caron S, Cotten A. Skull vault lesions: a review. AJR Am J Roentgenol 2015;205:840-7.
3 Turner RC, Lucke-Wold BP, Hwang R, et al. Lung cancer metastasis presenting as a solitary skull mass. J Surg Case Rep 2016;2016(6). doi:10.1093/jscr/rjw116

4 Guo X, Yin J, Jiang Y. Solitary skull metastasis as the first symptom of hepatocellular carcinoma: case report and literature review. Neuropsychiatr Dis Treat 2014; 10:681-6. 